OPEN ACCESS

Edited by:

Rainer Kurmayer,

University of Innsbruck, Austria

Reviewed by:

Karl Forchhammer,

Universität Tübingen, Germany

Vicente Mariscal,

Instituto de Bioquímica Vegetal y

Fotosintesis (IBVF), Spain

*Correspondence:

Cheng-Cai Zhang

cczhang@ihb.ac.cn

Specialty section:

This article was submitted to

Aquatic Microbiology,

a section of the journal

Frontiers in Microbiology

Received: 15 December 2017

Accepted: 09 April 2018

Published: 24 April 2018

Citation:

Zhang J-Y, Lin G-M, Xing $W-Y$ and Zhang C-C (2018) Diversity of Growth

Patterns Probed in Live

Cyanobacterial Cells Using

a Fluorescent Analog of a

Peptidoglycan Precursor.

Front. Microbiol. 9:791.

doi: 10.3389/fmicb.2018.00791

\section{Diversity of Growth Patterns Probed in Live Cyanobacterial Cells Using a Fluorescent Analog of a Peptidoglycan Precursor}

\author{
Ju-Yuan Zhang ${ }^{1}$, Gui-Ming Lin ${ }^{1}$, Wei-Yue Xing ${ }^{1,2}$ and Cheng-Cai Zhang ${ }^{1 *}$ \\ ${ }^{1}$ Key Laboratory of Algal Biology, Institute of Hydrobiology, Chinese Academy of Sciences, Wuhan, China, ${ }^{2}$ University of \\ Chinese Academy of Sciences, Beijing, China
}

Cyanobacteria were the first oxygenic photosynthetic organisms during evolution and were ancestors of plastids. Cyanobacterial cells exhibit an extraordinary diversity in their size and shape, and bacterial cell morphology largely depends on the synthesis and the dynamics of the peptidoglycan (PG) layer. Here, we used a fluorescence analog of the PG synthesis precursor D-Ala, 7-Hydroxycoumarin-amino-D-alanine (HADA), to probe the PG synthesis pattern in live cells of cyanobacteria with different morphology. They displayed diverse synthesis patterns, with some strains showing an intensive HADA incorporation at the septal region, whereas others gave an HADA signal distributed around the cells. Growth zones covering several cells at the tips of the filament were present in some filamentous strains such as in Arthrospira. In Anabaena PCC 7120, which is capable of differentiating heterocysts for $\mathrm{N}_{2}$ fixation, $\mathrm{PG}$ synthesis followed the cell division cycle. In addition, an HADA incorporation was strongly activated from 12 to $15 \mathrm{~h}$ following the initiation of heterocyst development, indicating a thickening of the PG layer in heterocysts. The PG synthesis pattern is diverse in cyanobacteria and responds to developmental regulation. The use of fluorescent analogs may serve as a useful tool for understanding the mechanisms of cell growth and morphogenesis operating in these organisms.

Keywords: cyanobacteria, peptidoglycan, growth pattern, cell wall, heterocyst, HADA

\section{INTRODUCTION}

Cyanobacteria are the only prokaryotes capable of oxygenic photosynthesis. Their occurrence on the Earth dates back to at least 2.45-2.32 Ga, but they may have first appeared even earlier, as evidenced by the fossils records (Bekker et al., 2004; Jeltsch, 2013). Cyanobacteria were responsible for the rise of oxygen levels, and certain ancient freshwater cyanobacteria were thought to be the ancestors of plastids through endosymbiosis with a heterotrophic host (Ponce-Toledo et al., 2017). During the long history of evolution, present-day cyanobacteria display a high degree of diversity in terms of morphology, living in the form of unicellular or filamentous strains, or in colonies. Depending on the strains, cyanobacterial cells also show a high diversity in their shape (round, spiral or rod), with sizes ranging from approximately $0.5 \mu \mathrm{m}$ in the case of marine 
Prochlorococcus to as large as 3-5 $\mu \mathrm{m}$ for freshwater Anabaena/Nostoc strains. How to account for this morphological diversity among cyanobacteria remains unclear compared to the understanding of other model organisms such as Escherichia coli and Bacillus subtilis.

One major element that contributes to the size and cell morphology in bacteria is the peptidoglycan (PG) layer (for a review, see Randich and Brun, 2015). The PG layer is a macromolecule that surrounds bacterial cells and maintains cell shape. It is composed of a polymer network of $\mathrm{N}$-acetylglucosamine and $\mathrm{N}$-acetylmuramic acid cross-linked with pentapeptide chains. Such networks are so rigid that isolated PG molecules (sacculi) are able to retain the shape of their original cells. Although rigid, the PG layer is highly dynamic and is constantly remodeled in order to keep pace with cell growth, division, or morphogenesis. Multiple modes of cell growth have been described in bacteria: lateral elongation, septation accompanying the process of cell constriction during cell division, polar elongation at the cell poles, or medial elongation emanating from the growth at the division plane (Randich and Brun, 2015). In one bacterium, such as E. coli, multiple modes of growth may together contribute to cell growth and morphogenesis. Two molecular machineries direct the synthesis of PG during cell cycle: the elongasome, responsible for insertion of new PG along the sidewall, and the divisome, which is located at the cell division plane. FtsZ treadmilling directs the dynamic synthesis of PG during cell constriction, whereas MreB coordinates the activity of the elongasome (Bisson-Filho et al., 2017; Yang et al., 2017). Both MreB and FtsZ are cytoskeletal elements (Busiek and Margolin, 2015) and are conserved in cyanobacteria (Zhang et al., 1995; Sakr et al., 2006; Hu et al., 2007).

Although the pentapeptides linking the sugar chains in PG may vary in composition depending on the bacteria, they usually contain D-Ala at the fourth and fifth positions. A fluorescent derivative of vancomycin (Van-FL), which tightly binds to the D-Ala-D-Ala dipeptide, has been used to trace PG biosynthesis sites and cell growth in a spectrum of bacteria (Tiyanont et al., 2006; Divakaruni et al., 2007; Lehner et al., 2013; Mariscal et al., 2016). Recently, several fluorescent D-amino acids (FDAAs) have been developed to probe the growth mode in bacterial cells by revealing the active part in a cell where PG synthesis occurs (Kuru et al., 2015). Van-FL is an analog of an antibiotic, whereas FDAAs, as analogs of native D-amino acids, can be incorporated into pentapeptide chains during PG synthesis with less influence on cell growth. Therefore, FDAAs can be used to trace PG synthesis in live cells. In Gram-negative prokaryotes, including cyanobacteria, the PG layer is within the periplasmic space, between the inner and outer membranes. In general, Gram-negative prokaryotes have a thinner PG layer compared to Gram-positive prokaryotes; however, cyanobacteria have a PG layer that is more reminiscent of those typically found in Gram-positive bacteria, such that it is thicker and has a higher degree of cross-linking (Hoiczyk and Hansel, 2000). Since little is known about the growth mode in cyanobacteria, we used one D-Ala analog, 7-hydroxycoumarin-3-carboxylic acidD-alanine (HADA), to probe the growth mode in cyanobacteria with different morphologies. HADA emits blue fluorescence, with little interference from the bright red fluorescence from the photosynthetic pigments. Our results reveal a great diversity in the mode of cell growth among cyanobacteria. We observed certain growth patterns that appeared to be unique. For example, the PG synthesis zone covered several cells at the tip of the filaments in some filamentous cyanobacteria. We also characterized the activity of PG synthesis in the heterocystforming cyanobacterium Anabaena PCC 7120 (also known as Nostoc PCC 7120), as an example of PG-layer remodeling during cell differentiation (Nicolaisen et al., 2009). Heterocysts, which are specialized in $\mathrm{N}_{2}$ fixation, are induced upon combined nitrogen starvation (Zhang et al., 2006; Herrero et al., 2016). Several genes encoding enzymes involved in PG metabolism have already been shown to be required for heterocyst development or functioning (Lázaro et al., 2001; Zhu et al., 2001; Lehner et al., 2011; Berendt et al., 2012; Videau et al., 2016; Bornikoel et al., 2017; Zheng et al., 2017). Our results showed an increased PG synthesis activity during heterocyst maturation, after the deposition of the polysaccharide layer, which led to a thick layer of PG surrounding the mature heterocyst. These results increase our understanding of the molecular mechanisms underlying PG synthesis and cellular morphogenesis in cyanobacteria.

\section{MATERIALS AND METHODS}

\section{Reagents}

Synthesis of HADA was carried out according to a previously described protocol (Kuru et al., 2015). HADA stock solution was prepared in DMSO at a concentration of $100 \mathrm{mM}$ and stored at $-20^{\circ} \mathrm{C}$ before use. Aztreonam was purchased from Sigma-Aldrich (Cat: PHR1785).

\section{Cyanobacterial Strains and Growth Conditions}

Anabaena PCC 7120 and Synechocystis PCC 6803 were maintained in our lab; Synechococcus elongatus PCC 7942, Arthrospira sp. FACHB 792, Spirulina subsalsa, Microcystis PCC 7806, and Oscillatoria animalis were obtained from Freshwater Algae Culture Collection at the Institute of Hydrobiology (FACHB); and Leptolyngbya sp. CB006, isolated from Tigris River in Baghdad City, was kindly provided by Ibrahim J. Abed in Baghdad University.

Arthrospira and Spirulina were cultivated in the Arthrospira medium described previously (Aiba and Ogawa, 1977). Anabaena, Oscillatoria, Leptolyngbya, Synechocystis, and Synechococcus elongtus were grown in BG11 medium (Stanier et al., 1971). Microcystis was grown in BG11 $1_{0}$ medium (BG11 without combined nitrogen) supplemented with $2 \mathrm{mM} \mathrm{NaNO}_{3}$ and $10 \mathrm{mM} \mathrm{NaHCO}$. All strains were grown axenically at $30^{\circ} \mathrm{C}$ in an incubator with the orbital shaking speed of $180 \mathrm{rpm}$ and the light density of $30 \mu \mathrm{mol} \mathrm{m}{ }^{-2} \mathrm{~s}^{-1}$.

To measure the growth of cyanobacterial strains, the fresh culture of each strain was inoculated into three 250-ml flasks with each flask containing $30 \mathrm{ml}$ of medium, to an initial 
optical density of 0.05 at $750 \mathrm{~nm}\left(\mathrm{OD}_{750}\right)$, and grown under conditions described above. The $\mathrm{OD}_{750}$ of each culture was measured every $12 \mathrm{~h}$ until the OD was 1.0. The growth curves were plotted using the 2-based logarithm of the $\mathrm{OD}_{750}$ reads (Y-axis) and sampling time (X-axis). The growth rate $(\mu)$ of each strain was calculated from the slope of the linear region (corresponding to the exponential growth) in the semilogarithmic curve. The generation time, or doubling time (d), was calculated using the equation: $d=1 / \mu$. The generation times of the strains were as follows: Synechocystis, Microcystis and S. elongatus, approximately 17 h; Oscillatoria, 28 h; Spirulina, 11 h; Arthrospira, 25 h; Leptolyngbya, 13 h; Anabaena, $20 \mathrm{~h}$.

\section{Construction of Plasmids and Strain}

The sequences of all the primers used in this study are listed in Supplementary Table S1. All the sequences of the plasmids used in this study have been deposited into GenBank.

The vector pCint2 (GenBank accession number: MH050934; Supplementary Figure S1) is a derivate of pRL271 (Cai and Wolk, 1990) with the erythromycin-resistant gene cassette deleted from the original plasmid. To construct pCint2, the vector pRL271 was amplified with the primers of PCINT2a and PCINT2b and the 5450 bp PCR fragment was circularized using the Vazyme ClonExpress II One Step Cloning Kit (Cat: C11201). A sequence encoding a protein linker, a cyanobacterial codon-optimized syfp2 ORF, a TwinStrep tag, and an artificial transcriptional terminator was synthesized by GenScript and cloned into the vector pUC57-simple (GenScript) via EcoRV, resulting in the plasmid pSYFP2 (GenBank accession number: MH050935; Supplementary Figure S2). To make pSYFP2sp (GenBank accession number: MH050936; Supplementary Figure S3), a spectinomycin/streptomycin resistance gene (1053 bp) was amplified from the omega fragment (Prentki and Krisch, 1984) using the primers of PspF214m and PspR797, and cloned into ApaI-linearized pSYFP2 using the Vazyme ClonExpress II One Step Cloning Kit. The plasmid pFtsZ-SYFP2 was constructed as follows. Two fts $Z$ regions were amplified from the chromosome of Anabaena PCC 7120 with the primer pairs of Palr3858F160/Palr3858R1284 (product size: 1167 bp) and Palr3858F1353/Palr3858R2377 (product size: $1062 \mathrm{bp}$ ), respectively. The YFP coding region together with the spectinomycin/streptomycin resistance gene (2124 bp) was amplified from the plasmid pSYFP2-sp with the primers of Psyfp2spF and Psyfp2spR. The three PCR fragments were inserted into PstI/Xho linearized pCint 2 using the Vazyme ClonExpress MultiS One Step Cloning Kit (Cat: C11301), resulting in pFtsZ-SYFP2 (GenBank accession number: MH050937; Supplementary Figure S4). All plasmids were verified by sequencing.

The plasmid pFtsZ-SYFP2 was transferred into wildtype Anabaena PCC7120 by conjugation to make the Anabaena FtsZSYFP translational fusion strain. The methods of conjugation and selection of desired colonies followed a previously described procedure (Cai and Wolk, 1990; Elhai et al., 1997). The genotype of the obtained strain was verified by PCR (data not shown). The translational fusion strain was grown in BG11 medium supplemented with $5 \mu \mathrm{g} \mathrm{ml}^{-1}$ spectinomycin and $2.5 \mu \mathrm{g} \mathrm{ml}^{-1}$ streptomycin.

\section{HADA Labeling}

For pulse labeling, exponentially growing cells were diluted to $\mathrm{OD}_{750}=0.2$ in a medium containing $800 \mu \mathrm{M}$ HADA and grown for $2 \sim 10 \%$ generation time, then washed with cold $1 \times$ phosphate buffered saline (PBS, which has a pH of 7.4 and contains $137 \mathrm{mM}$ $\mathrm{NaCl}, 2.7 \mathrm{mM} \mathrm{KCl}, 8 \mathrm{mM} \mathrm{Na} 2 \mathrm{HPO}_{4}$, and $\left.14.6 \mathrm{mM} \mathrm{KH}_{2} \mathrm{PO}_{4}\right)$ and imaged immediately.

For longer labeling times, exponentially growing cells were diluted to $\mathrm{OD}_{750}=0.2$ in media containing $200 \mu \mathrm{M}$ HADA and grown for $1 \sim 2$ generation times. For heterocyst labeling, $200 \mu \mathrm{M}$ HADA was added into BG $11_{0}$ medium at different time points. HADA-labeled cells were washed either with the growth medium to remove free HADA from the culture and allowed to grow without HADA, or with cold $1 \times$ PBS and imaged immediately.

For HADA labeling after cell division inhibition, the FtsZYFP translational fusion strain exponentially growing in BG11 was treated with $100 \mu \mathrm{M}$ aztreonam for $36 \mathrm{~h}$, followed by the combined treatment of $100 \mu \mathrm{M}$ aztreonam and $200 \mu \mathrm{M}$ HADA for $24 \mathrm{~h}$.

\section{Microscopy and Image Processing}

HADA has fluorescence properties similar to blue fluorescent proteins (BFPs), with a maximal excitation wavelength at $405 \mathrm{~nm}$ and the maximal emission at $460 \mathrm{~nm}$ (Kuru et al., 2015). The Nikon filter sets of BFP (EX379-401, DM420LP, EM435-485), B-2A (EX450-490, DM515LP, EM515LP), and YFP HYQ (EX490510, DM520-580, EM520-550) were used to image HADA fluorescence, the fluorescence of photosynthetic pigments, and FtsZ-YFP fluorescence, respectively. All microscopic images were taken using a Nikon Ti-E inverted fluorescence microscope equipped with a Plan Apo $100 \times / 1.70$ Oil Ph3 DM objective lens, an HQ2 CCD camera. All HADA pictures were taken with the same exposure time (1 s). Merging of image channels, straightening of spiral or curved cyanobacterial filaments, and image quantification were performed with ImageJ 1.50 (Schneider et al., 2012).

\section{RESULTS}

\section{Cell Growth of Unicellular Strains Depends on Major Septal and Minor Peripheral PG Synthesis (Synechocystis and S. elongatus) or Medial Elongation (Microcystis)}

We tested the effect of different HADA concentrations on the growth of strains used in this study and found that a concentration of $200 \mu \mathrm{M}$ had little effect, whereas a concentration up to 400 or $800 \mu \mathrm{M}$ slightly impeded cell growth, making cells yellowish (Supplementary Figure S5). Thus, we used $200 \mu \mathrm{M}$ for long-term labeling and $800 \mu \mathrm{M}$ for pulse labeling for half an hour or one hour, following the rules that pulse-labeling times correspond to $2-5 \%$ of a generation time, and long-term 
labeling times correspond to 1-2 generation times (Kuru et al., 2015). The doubling time of the strains used in this study varied between 11 and $28 \mathrm{~h}$ under our culture conditions.

We first tested the synthesis of the PG layer in two model unicellular strains, representing each a distinct cellular morphology of cyanobacteria. Synechocystis is round-shaped, whereas S. elongatus is rod-shaped. Both strains have a binary division mode, with a generation time of approximately $17 \mathrm{~h}$ under our culture condition. We first performed pulse labeling for $30 \mathrm{~min}$ with $800 \mu \mathrm{M}$ HADA (Figure 1A). In both strains, intensive incorporation of HADA occurred at the mid site, starting from young cells before any sign of cell constriction, until the very end of the cell cycle when cell constriction was almost finished. Occasionally, HADA labeling at one cell pole could be observed, due to the separation of two daughter cells during the course of HADA pulse labeling. Thus, both strains, with different cell shapes, showed substantial incorporation of HADA at the septal region. We also performed continuous HADA labeling for up to $24 \mathrm{~h}$ with $200 \mu \mathrm{M}$ HADA (Figure 1B). For both strains, in addition to intensive septal labeling, weaker peripheral HADA fluorescence could also be seen, indicating that these strains had a low peripheral PG synthesis activity. For Synechocystis, a new division plane positioned perpendicularly to the older one could often be found in one or both daughter cells before they separated. This finding is consistent with the division pattern of this strain (Marbouty et al., 2009).

We also studied the PG synthesis pattern in Microcystis PCC 7806 which is a round-shaped strain known for its production of the toxin microcystin (Dittmann and Wiegand, 2006). Microcystis and Synechocystis showed no obvious difference in HADA incorporation when pulse-labeled for $30 \mathrm{~min}$ with $800 \mu$ M HADA (data not shown). Like Synechocystis, Microcystis also showed a septal and peripheral HADA incorporation with continuous HADA labeling for $12 \mathrm{~h}$ at a concentration of $200 \mu \mathrm{M}$ (Figure 1B). Interestingly, a labeling pattern of double stripe at the mid cell position could be found in many cells (indicated by arrows in Figure 1B). Such a growth pattern has been reported in some bacteria, called preseptal or medial elongation, corresponding to PG synthesis bordering the division plane before full assembly of the divisome (Randich and Brun, 2015). Thus, unicellular cyanobacterial strains adopted different strategies for PG synthesis during cell growth.

\section{The Filamentous Cyanobacteria Arthrospira and Oscillatoria Depend on Both Septal and Peripheral PG Synthesis for Cell Growth}

We used two filamentous cyanobacteria, which do not fix nitrogen, as examples to examine the cell growth pattern by HADA labeling. The cells of both strains have a disc shape growing much shorter than wide (Figure 2). For Arthrospira, we first performed a short-time pulse labeling of $30 \mathrm{~min}$ with $800 \mu \mathrm{M}$ of HADA. At the tip of many filaments, a high labeling zone, covering several cells could be observed (Figure 2A), and

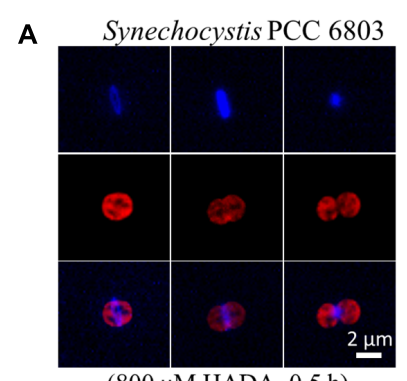

(800 $\mu \mathrm{M}$ HADA, $0.5 \mathrm{~h})$

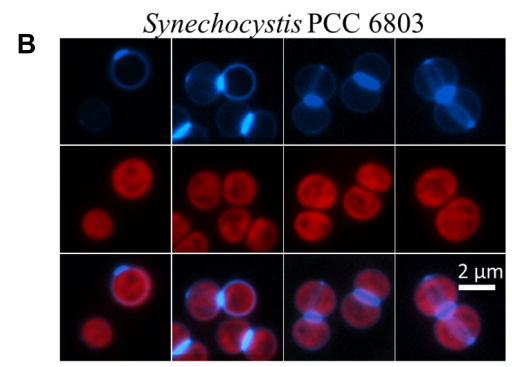

(200 $\mu \mathrm{M}$ HADA, $24 \mathrm{~h})$

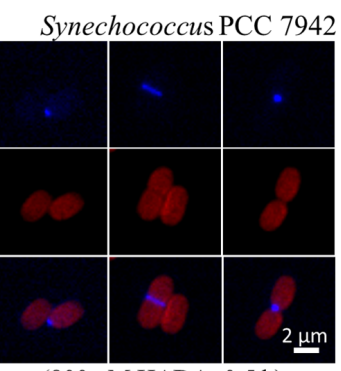

$(800 \mu \mathrm{M}$ HADA, $0.5 \mathrm{~h})$

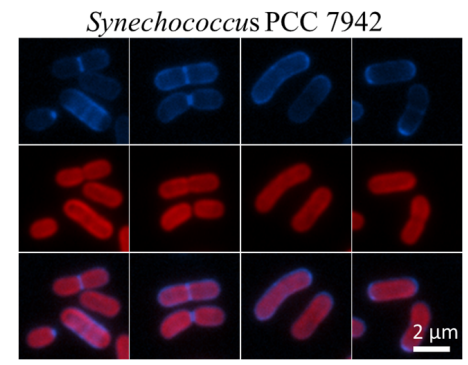

(200 $\mu \mathrm{M}$ HADA, 24 h)

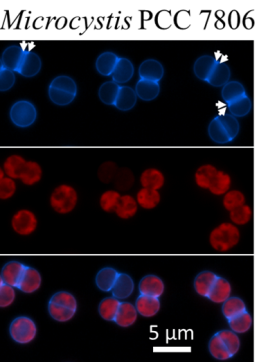

$(200 \mu \mathrm{M}$ HADA, $12 \mathrm{~h})$

FIGURE 1 | HADA labeling in three unicellular cyanobacteria: Synechocystis PCC 6803, Synechococcus elongatus PCC 7942 , and Microcystis PCC 7806. (A) Pulse labeling of Synechocystis and Synechococcus elongatus with a high concentration of HADA (800 $\mu$ M) for 30 min, followed by removal of HADA by washing and observation by fluorescence microscopy. (B) Continuous labeling with a low concentration of HADA in Synechocystis, Synechococcus elongatus, and Microcystis. For Synechocystis and Synechococcus elongatus, three or four sets of photos are shown, and each demonstrates a representative HADA labeling pattern. HADA signal is in blue, and photosynthetic pigment in red, and the overlay for the images of same cells acquired through the two channels. Arrows indicates the pre-septal division mode in Microcystis. 
A

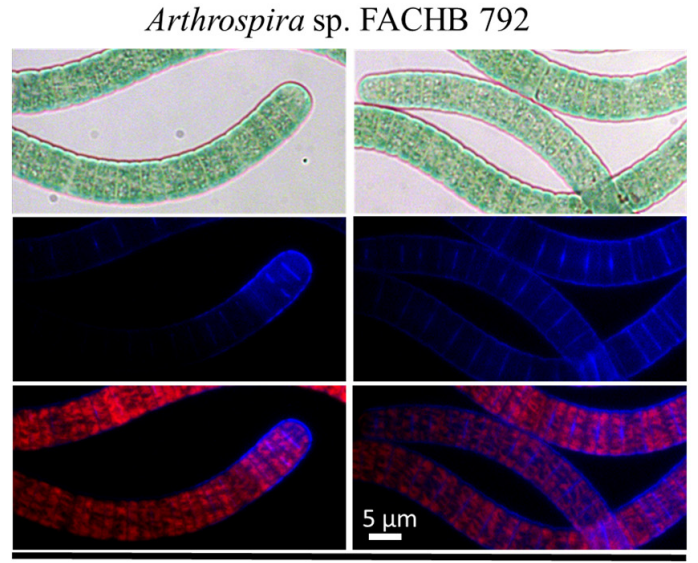

$(800 \mu \mathrm{M}$ HADA, $0.5 \mathrm{~h})$

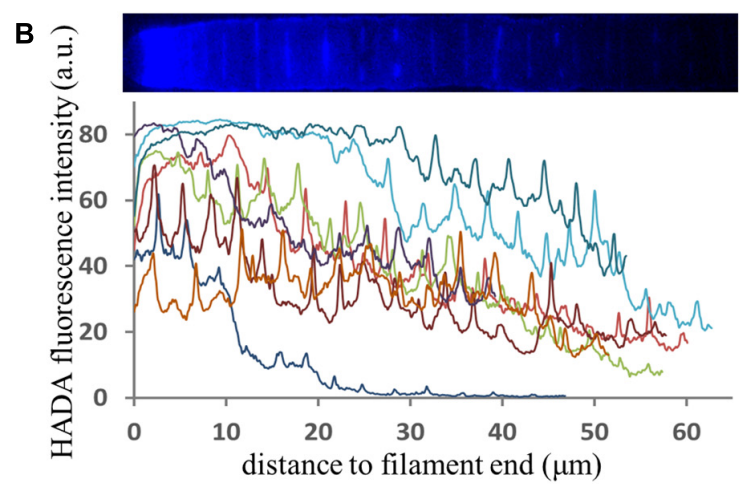

C Arthrospira sp. FACHB 792
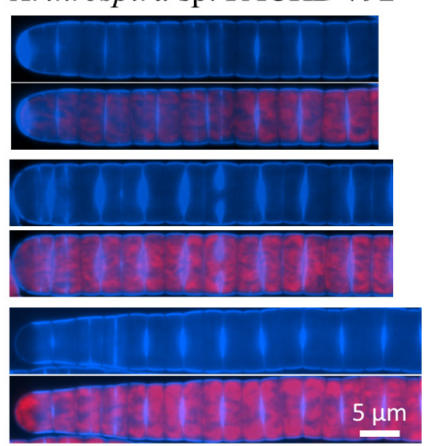

(200 $\mu$ M HADA, 24 h)

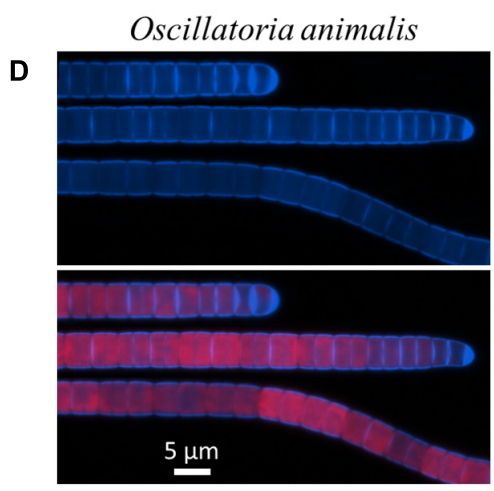

(200 $\mu \mathrm{M}$ HADA, 24 h)

FIGURE 2 | Growth pattern of the filamentous cyanobacteria Arthrospira sp. FACHB 792 and Oscillatoria animalis. (A,C) Arthrospira pulse-labeled with $800 \mu$ M of HADA for half an hour or long-term labeled with $200 \mu \mathrm{M}$ of HADA for $24 \mathrm{~h}$. (B) The HADA signal at the tips of some pulse labeled Arthrospira quantified with Image J. Every curve in the graph represents the fluorescence intensity along one end of a filament as exemplified above the graph. The filaments of long-term labeled Arthrospira were straightened with ImageJ. (D) Oscillatoria incubated with $200 \mu \mathrm{M}$ of HADA for $24 \mathrm{~h}$. Bright-field photos (gray background pictures in panel A), as well as images of HADA fluorescence (blue), photosynthetic pigment fluorescence (red), and their merges are shown.

quantification of the HADA signal revealed stronger PG synthesis at the end of these filaments (Figure 2B). In general, both septal and peripheral PG synthesis could be seen, indicating that the cell growth requires both lateral and septal PG synthesis during cell growth, although much stronger HADA labeling occurred at the newly formed division site just separating two daughter cells (Figure 2C). Oscillatoria displayed a similar PG synthesis pattern as Arthrospira (Figure 2D), with both a lateral and septal HADA incorporation.

Similar pattern of PG synthesis could also be observed with a Leptolyngbya species (with rod-shaped cells along the filaments) and a Spirulina species (twisted cells along the filaments); although the peripheral HADA signal in these species appeared weaker than in Oscillatoria and Arthrospira (Figure 3). HADA pattern in Spirulina was twisted along the filament, mirroring the shape of the filaments. The Leptolyngbya species grew with a doubling time of approximately $13 \mathrm{~h}$ but required a much longer incubation time to visualize HADA incorporation (3 days). At $24 \mathrm{~h}$ after transferring cells into HADA-free medium, HADA fluorescence at the septal sites mostly still remained, whereas those at the peripheral disappeared, indicating that PG turnover was faster at the cell periphery than at the cell division site in this strain.

\section{Cell Growth and Division in the $\mathrm{N}_{2}$-Fixing Filamentous Strain Anabaena}

Anabaena PCC 7120 is a model strain for the studies of heterocyst development. We thus used this organism to trace the pattern of PG synthesis during both vegetative growth and heterocyst development. We first incubated filaments of Anabaena with various concentrations of HADA for different times and found that labeling time contributed to labeling efficiency much more than HADA concentration. For instance, $3 \mathrm{~h}$ of pulse labeling only gave a dim HADA signal at a few cell junctions irrespective of the used concentration of HADA, whereas $9 \mathrm{~h}$ of incubation with $200 \mu \mathrm{M}$ HADA could gave bright labeling (data not shown). Therefore, we used a period of 9-24 h, for efficient labeling in Anabaena in subsequent experiments with $200 \mu \mathrm{M}$ of HADA. Under vegetative growth, an intensive HADA signal could be detected in young cells at the division site, or old cells already finished cell constriction; at the same time, a weak but 


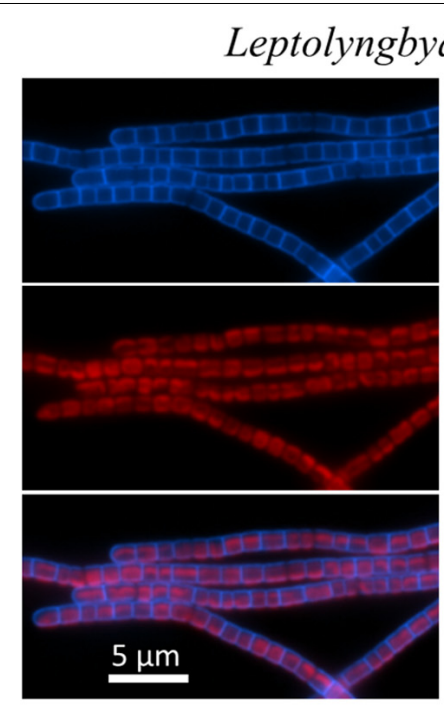

(200 $\mu \mathrm{M}$ HADA, $3 \mathrm{~d})$
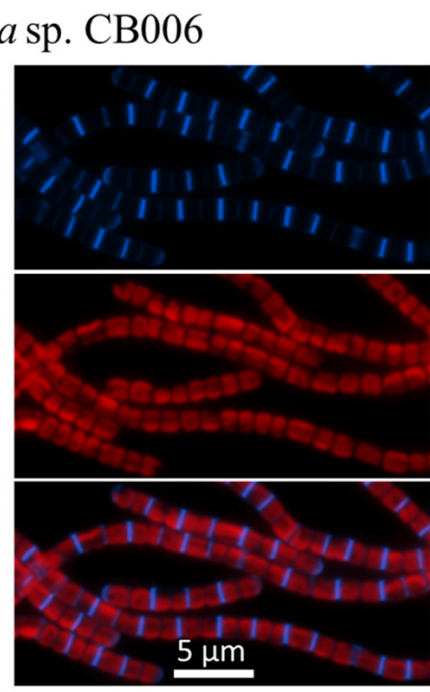

$(200 \mu \mathrm{M}$ HADA for $3 \mathrm{~d}$, then no HADA for $24 \mathrm{~h}$ )

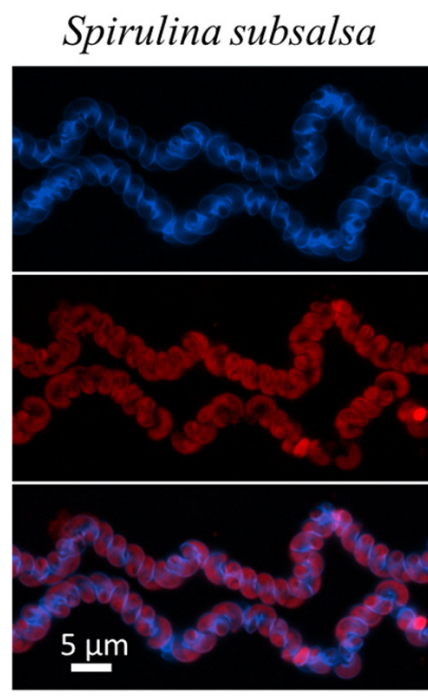

(200 $\mu \mathrm{M}$ HADA, 24 h)

FIGURE 3 | HADA incorporation into Leptolyngbya sp. CB006 and Spirulina subsalsa. Leptolyngbya requires a longer incubation time with HADA. Photos of HADA fluorescence (blue), photosynthetic pigment fluorescence (red), and their merges are shown.

detectable HADA signal at the cell periphery could also be seen (Figure 4A).

FtsZ, as the cell division initiating protein, assembles into Z-ring at the mid cell during the early stage of cell division. To explore the relationship between cell division and PG synthesis, we first probed the PG synthesis with HADA labeling in a strain in which FtsZ is fused to YFP (Figure 5). Three types of labeling could be observed. First, there were cells containing a Z-ring at the mid-cell position, but no detectable incorporation of HADA (red arrows, Figure 5A) or sign of cell constriction were observed. Second, both FtsZ-YFP and HADA fluorescence could be observed at the mid-cell position, with the two fluorescence signals superimposed (white arrows). Some of these cells did not show evidence of cell constriction, whereas others already evinced signs of cell constriction, indicating that cell division progressed to the constriction stage. Finally, some cells in which cell division was completed, with a narrow cell constriction between two cells, gave only a HADA signal but no FtsZ-YFP fluorescence (asterisks). At the end of the cell division, the Z-ring disassembled at the division site, but HADA incorporated at the constriction site remained for a long time, indicating a slow turnover of PG at the septa. Consistent with these observations, even after $72 \mathrm{~h}$ of HADA removal, HADA fluorescence could still be observed at many cell-cell junctions (data not shown). The antibiotic aztreonam targets the cell division protein FtsI/PBP3 which is involved in PG synthesis at the bacterial division site. In the presence of aztreonam, cell division could be impaired, leading to elongated forms of cells. Under such conditions, FtsZ-YFP could still be observed as a ring structure, but a HADA incorporation at the peripheral sidewall became obvious (Figure 5B).

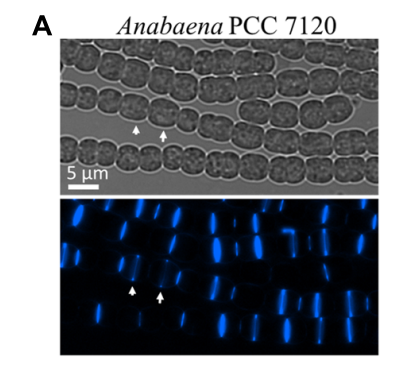

(BG11, $200 \mu \mathrm{M}$ HADA, 24 h)

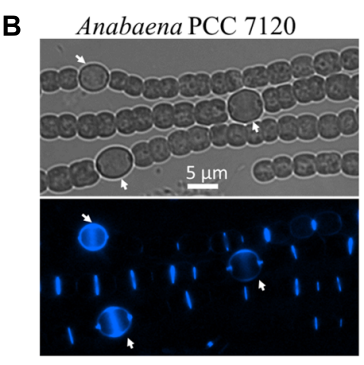

(BG11, $200 \mu \mathrm{M}$ HADA, 24 h)
FIGURE 4 | PG synthesis during vegetative growth and heterocyst development in Anabaena PCC 7120. Filaments of Anabaena were grown in BG11 using nitrate as a combined nitrogen source, then incubated with $200 \mu \mathrm{M}$ of HADA in BG11 (A), or in BG110 (B) to induce heterocyst development. Arrows in $\mathrm{A}$ indicate elongated cells that just started to incorporate HADA at the division site, and in B heterocysts. Bright-field (gray) and HADA fluorescence (blue) images are shown.

\section{Regulation of PG Synthesis During Heterocyst Development}

As shown above, PG synthesis in vegetative cells was mainly at the division or constriction site. However, as terminally differentiated cells developed from vegetative cells, heterocysts are incapable of cell division. To explore whether PG synthesis is still active in heterocysts, we induced heterocysts by growing vegetative filaments of Anabaena in a nitrogen-free medium containing $200 \mu \mathrm{M}$ HADA, and measured HADA fluorescence in mature heterocysts after $24 \mathrm{~h}$ of induction. As shown in Figure 4B, intensive HADA incorporation in heterocysts was observed at 


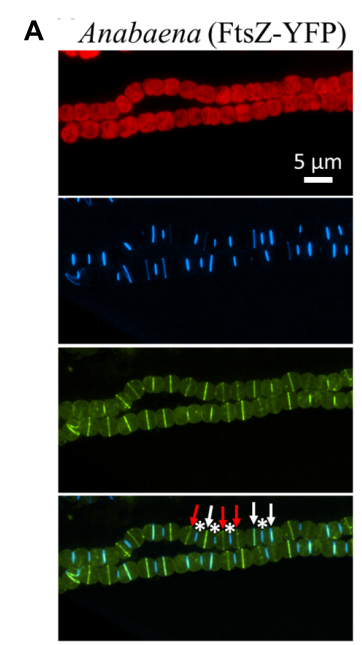

$(200 \mu \mathrm{M}$ HADA, $24 \mathrm{~h})$
B

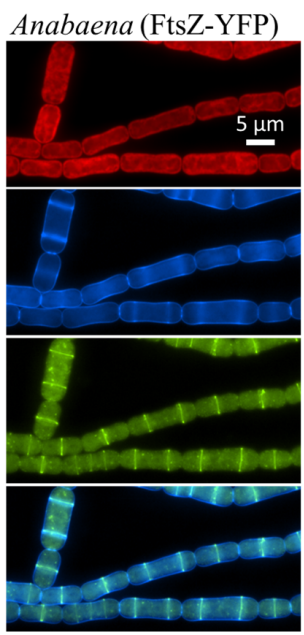

$(100 \mu \mathrm{M} \mathrm{Az}$ for $36 \mathrm{~h}$, then $100 \mu \mathrm{MAz}$ and $200 \mu \mathrm{M}$ HADA for $24 \mathrm{~h}$ )

FIGURE 5 | Relationship between cell division and PG synthesis in Anabaena PCC 7120. (A) An Anabaena strain in which the cell division gene fts $Z$ was replaced by a ftsZ-yfp translational fusion was treated with HADA, and imaged under a fluorescence microscope. The top panel shows red photosynthetic pigment and the HADA signal in blue; the middle panel shows the Z-ring in yellow; and the bottom panel shows a superimposition of the same filaments with both HADA and FtsZ-YFP signals. Asterisks indicate cell-cell junctions labeled by HADA, white arrows indicate septa having both HADA signal and FtsZ-YFP signal, and red arrows indicate septa having FtsZ-YFP signal only. (B) The same recombinant strain as in A was treated with aztreonam (Az), an antibiotic targeting to Ftsl, a PG synthase involved in cell division. Cells elongated as a consequence of cell division inhibition as previously reported (Sakr et al., 2006). The same filaments were pictured in red (photosynthetic pigments), blue (HADA), yellow (FtsZ-YFP) and superimposition of the HADA and YFP signals.

the central peripheral areas, as well as at the polar regions, with PG labeling protruding to neighboring vegetative cells. Overall, HADA fluorescence intensity was much stronger in mature heterocysts than in vegetative cells. Because PG synthesis appeared to be upregulated in heterocysts, we determined the timing of PG synthesis during heterocyst development (Figure 6). Alcian blue is a dye that can specifically stain the heterocyst polysaccharide layer, even at the early phase of heterocyst development before any morphological signs. As shown in Figure 6A, whereas no Alcian blue staining could be observed in filaments $7 \mathrm{~h}$ after the initiation of heterocyst development, some cells were already weakly stained at $8 \mathrm{~h}$, indicating that the polysaccharide layer began to be deposited at the cell wall. From $10 \mathrm{~h}$ on, clear Alcian blue staining could be observed in the developing cells, indicative of proheterocyst formation. At $12 \mathrm{~h}$, very few proheterocysts gave rise to HADA fluorescence, suggesting that some had begun to synthesize PG at the cell wall. At $15 \mathrm{~h}$, all proheterocysts showed increased HADA incorporation, indicating that active PG synthesis occurred in these cells. This observation was consistent with the quantification of the HADA signal in a representative number of differentiating cells at these time points (Figure 6B).

\section{DISCUSSION}

In this study, we investigated the PG synthesis patterns by HADA staining in several cyanobacterial strains which cover most of the common morphological types known in cyanobacteria: unicellular, filamentous, spiral, or heterocystous. We also tested several strains that grow in colonial or branchingfilamentous forms, but the existence of a gelatinous sheath surrounding the cells prevented a proper staining of PG by HADA incorporation (data not shown). Most of the commonly found cell morphologies in cyanobacteria are represented in this study. Despite the diversity of cell size and morphology, the PG synthesis patterns observed in cyanobacteria share some common features and may display a distinct characteristic depending on the strain examined. First, as a general rule, all the cyanobacterial strains we examined thus far showed intensive HADA incorporation at the septal site. Once incorporated at the division site, HADA remained for a long time after transferring cells into HADA-free medium, which is evidence of a slow turnover. Second, peripheral PG synthesis (or lateral elongation) was also common in cyanobacteria, but varied extensively: some strains such as Leptolyngbya required a long incubation with HADA to reveal the lateral PG synthesis, whereas other strains such as Synechocystis or Anabaena gave an HADA signal distributed around the cell at a similar intensity (see Arthrospira, Leptolyngbya, and Oscillatoria, for example). HADA signal intensity may correlate with the degree of septation after cell division, (i.e., the degree of polar morphological remodeling). Indeed, when the two daughter cells were either completely separated after cell division (unicellular strains such as Synechocystis for example), or cell constriction was severe with a much narrower connection at cell-cell junctions, the cell poles generated during cell division required extensive PG hydrolysis and resynthesis in order to be remodeled. In contrast, in those strains whose cell constriction was limited, PG synthesis was restricted mainly to the separation of the two daughter cells. Beyond these general rules of PG synthesis, some cyanobacterial strains displayed distinct features: Microcystis had a preseptaltype growth mode, similar as that observed in Caulobacter crescentus (Aaron et al., 2007); Arthrospira and Oscillatoria filaments often displayed a stronger, or even graded HADA signal intensity at the tip of the filaments. Thus, cyanobacteria show great diversity in term of cell growth mode.

Using Anabaena, we examined PG synthesis in vegetative cells and its relationship to cell division. The data suggested that HADA incorporation and consequently, PG synthesis, occurred at the division site from the onset until polar morphogenesis, as well as weakly along the long axis of the cell wall (Figure 4A). In some larger vegetative cells, corresponding to those ready to divide, a faint line of HADA fluorescence at the mid-cell position could be seen before cell constriction (white arrows in Figure 4A), suggesting that the PG synthesis at the division site starts relatively early during the cell cycle. Furthermore, synthesis of PG at the division site likely followed the direction of FtsZ. This was suggested by the evidence that only a Z-ring, but no HADA signal, was found at the septum in many cells without constriction formation, whereas a Z-ring superimposed with 
A

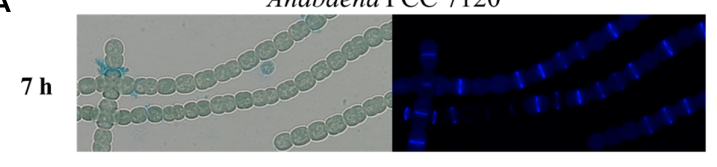

8 h

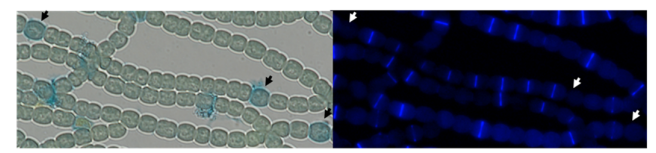

$10 \mathrm{~h}$
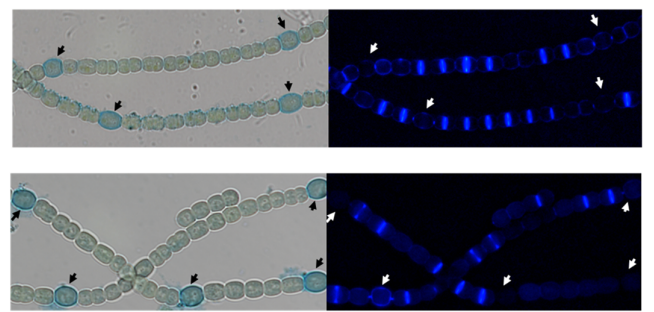

$15 \mathrm{~h}$

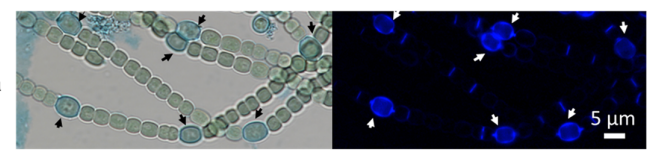

B

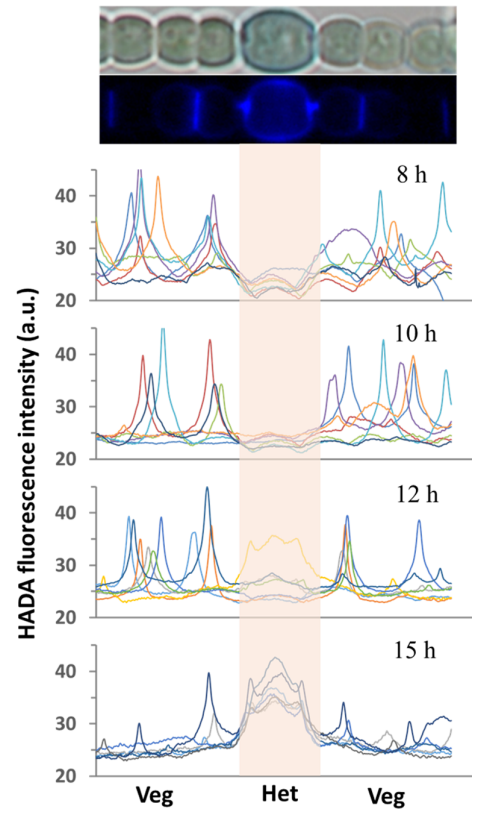

FIGURE 6 | Determination of the timing of PG synthesis in heterocysts. Hetercoysts differentiation takes approximately 20-24 h after the deprivation of combined nitrogen from the growth medium. Heterocysts were induced in the BG110 medium containing $200 \mu \mathrm{M}$ HADA. Seven hours after the induction, filaments were stained with Alcian blue which binds to heterocyst-specific polysaccharide layer, to better recognize the developing cells (arrows). (A) The filaments were examined under both bright-field and for HADA fluorescence to determine the incorporation of HADA into developing heterocysts. The same procedure was followed after $10 \mathrm{~h}, 12 \mathrm{~h}$ and $15 \mathrm{~h}$ of induction. All HADA fluorescence images were taken using the same exposure time (1 s) and were processed with the same procedures using ImageJ. (B) Quantification of HADA incorporation in developing and mature heterocysts at $8 \mathrm{~h}, 10 \mathrm{~h}, 12 \mathrm{~h}$, and $15 \mathrm{~h}$. At each time point, the fluorescence signal along seven representative short filaments, each of which consists of one heterocyst (Het) at the center and a few vegetative cells (Veg) on each side, were quantified with ImageJ. Every curve in the graphs represents the fluorescence intensity along one filament. The signal from developing or mature heterocysts in the graphs is indicated by the center shadow area. The bright-field microscopy image and the HADA fluorescence picture of one filament from the $15 \mathrm{~h}$ sample were shown at the top of the panel for illustration purpose.

HADA signal appeared in all cells that had begun constricting (Figure 5A). The superimposition of the Z-ring and HADA incorporation activity at cell septum implies cell division and HADA incorporation are highly coordinated. When cell division was inhibited by aztreonam treatment, cells became large and elongated. In most of these cells, HADA incorporation was no longer restricted to the $\mathrm{Z}$-ring site. Rather, it occurred on the entire cell surface (Figure 5B). Thus, PG synthesis and cell division can be uncoupled at the septum when cell division is blocked.

Heterocysts are differentiated from vegetative cells upon combined nitrogen deprivation. Compared to vegetative cells, heterocysts are larger and feature a thick cell envelope with two additional layers: an inner glycolipid layer and an outer polysaccharide layer (Wolk, 1996). We found that PG synthesis in differentiating cells (proheterocysts) became active after the formation of the polysaccharide layer, at a relatively late stage of heterocyst development (Figure 6). HADA labeling occurred around the whole proheterocyst cells, in contrast to vegetative cells, where labeling was mainly noted at the division site. The HADA signal in proheterocysts covered a large central area, which corresponds to a typical lateral growth mode found in E. coli or B. subtilis. This is also similar to the elongation mode of vegetative cells when septal PG synthesis is inhibited by aztreonam. The cell division protein FtsZ is known to be downregulated during heterocyst development at both transcriptional and post-transcriptional levels (Kuhn et al., 2000; Wang and $\mathrm{Xu}, 2005)$. Thus, the PG synthesis pattern in proheterocysts is likely to be independent of FtsZ but dependent on MreB, which has been reported to be upregulated in proheterocysts (Hu et al., 2007). The regulation of PG synthesis is consistent with the finding that several PG synthesis enzymes are required for heterocyst development or functioning (Lázaro et al., 2001; Zhu et al., 2001; Lehner et al., 2011; Berendt et al., 2012; Videau et al., 2016; Bornikoel et al., 2017; Zheng et al., 2017). Based on our findings and previous reports, we propose that a thick PG layer is another characteristic of mature heterocysts, in addition to their glycolipid and polysaccharide layers. Additionally, some of the enzymes involved in PG metabolism may affect the functioning of heterocysts by modulating PG synthesis during heterocyst maturation, in addition to their roles in cell-cell communication.

The fluorescent derivative of vancomycin, Van-FL, was previously used to stain PG in the heterocyst-forming cyanobacteria Anabaena PCC 7120 and Nostoc punctiforme (Lehner et al., 2013; Rudolf et al., 2015; Mariscal et al., 2016). The staining of vegetative cells with Van-FL showed similar patterns to that of HADA, with intensive septal labeling. 
However, the Van-FL signal did not increase in heterocysts, which differs from our observations using HADA in this study. Two reasons may contribute to this difference. First, Van-FL is an analog of the antibiotic vancomycin. Thus, it is unsuitable to follow the entire process of heterocyst development, which lasts for more than $20 \mathrm{~h}$, with live-cell imaging. Second, HADA incorporation occurs at the late stage of heterocyst development and Van-FL is a relatively large molecule (1921 Da) compared to HADA (292 Da). Thus, it is more difficult for Van-FL to penetrate the already formed thick proheterocyst envelope and outer membrane to reach PG synthesis sites during the staining procedure. In conclusion, HADA is a more suitable labeling tool for the study of heterocyst development.

\section{AUTHOR CONTRIBUTIONS}

C-CZ, J-YZ, and G-ML designed the study and analyzed the data. J-YZ, G-ML, and W-YX performed the experiments. C-CZ and J-YZ wrote the manuscript.

\section{REFERENCES}

Aaron, M., Charbon, G., Lam, H., Schwarz, H., Vollmer, W., and Jacobs-Wagner, C. (2007). The tubulin homologue FtsZ contributes to cell elongation by guiding cell wall precursor synthesis in Caulobacter crescentus. Mol. Microbiol. 64, 938-952. doi: 10.1111/j.1365-2958.2007.05720.x

Aiba, S., and Ogawa, T. (1977). Assessment of growth yield of a blue - green alga, Spirulina platensis, in axenic and continuous culture. Microbiology 102, 179-182. doi: 10.1099/00221287-102-1-179

Bekker, A., Holland, H. D., Wang, P. L., Rumble, D., Stein, H. J., Hannah, J. L., et al. (2004). Dating the rise of atmospheric oxygen. Nature 427, 117-120. doi: 10.1038/nature02260

Berendt, S., Lehner, J., Zhang, Y. V., Rasse, T. M., Forchhammer, K., and Maldener, I. (2012). Cell wall amidase AmiC1 is required for cellular communication and heterocyst development in the cyanobacterium Anabaena PCC 7120 but not for filament integrity. J. Bacteriol. 194, 5218-5227. doi: 10.1128/JB.00912-12

Bisson-Filho, A. W., Hsu, Y. P., Squyres, G. R., Kuru, E., Wu, F., Jukes, C., et al. (2017). Treadmilling by FtsZ filaments drives peptidoglycan synthesis and bacterial cell division. Science 355, 739-743. doi: 10.1126/science.aak9973

Bornikoel, J., Carrión, A., Fan, Q., Flores, E., Forchhammer, K., Mariscal, V., et al. (2017). Role of two cell wall amidases in septal junction and nanopore formation in the multicellular cyanobacterium Anabaena sp. PCC 7120. Front. Cell. Infect. Microbiol. 7:386. doi: 10.3389/fcimb.2017.00386

Busiek, K. K., and Margolin, W. (2015). Bacterial actin and tubulin homologs in cell growth and division. Curr. Biol. 25, R243-R254. doi: 10.1016/j.cub.2015.01.030

Cai, Y. P., and Wolk, C. P. (1990). Use of a conditionally lethal gene in Anabaena sp. strain PCC 7120 to select for double recombinants and to entrap insertion sequences. J. Bacteriol. 172, 3138-3145. doi: 10.1128/jb.172.6.3138-3145.1990

Dittmann, E., and Wiegand, C. (2006). Cyanobacterial toxins-occurrence, biosynthesis and impact on human affairs. Mol. Nutr. Food Res. 50, 7-17. doi: 10.1002/mnfr.200500162

Divakaruni, A. V., Baida, C., White, C. L., and Gober, J. W. (2007). The cell shape proteins $\mathrm{MreB}$ and $\mathrm{MreC}$ control cell morphogenesis by positioning cell wall synthetic complexes. Mol. Microbiol. 66, 174-188. doi: 10.1111/j.1365-2958. 2007.05910.x

Elhai, J., Vepritskiy, A., Muro-Pastor, A. M., Flores, E., and Wolk, C. P. (1997). Reduction of conjugal transfer efficiency by three restriction activities of Anabaena sp. strain PCC 7120. J. Bacteriol. 179, 1998-2005.

Herrero, A., Stavans, J., and Flores, E. (2016). The multicellular nature of filamentous heterocyst-forming cyanobacteria. FEMS Microbiol. Rev. 40, 831-854. doi: 10.1093/femsre/fuw029

\section{FUNDING}

The work was funded by the Key Research Program of Frontier Sciences of the Chinese Academy of Sciences (Grant No. QYZDJ-SSW-SMC016), and the Recruitment Program of Global Experts of China (Grant No. Y523011).

\section{ACKNOWLEDGMENTS}

The authors thank the handling editor and the reviewers for their critical reading of the manuscript and helpful suggestions.

\section{SUPPLEMENTARY MATERIAL}

The Supplementary Material for this article can be found online at: https://www.frontiersin.org/articles/10.3389/fmicb. 2018.00791/full\#supplementary-material

Hoiczyk, E., and Hansel, A. (2000). Cyanobacterial cell walls: news from an unusual prokaryotic envelope. J. Bacteriol. 182, 1191-1199. doi: 10.1128/JB.182.5.11911199.2000

Hu, B., Yang, G., Zhao, W., Zhang, Y., and Zhao, J. (2007). MreB is important for cell shape but not for chromosome segregation of the filamentous cyanobacterium Anabaena sp. PCC 7120. Mol. Microbiol. 63, 1640-1652. doi: 10.1111/j.1365-2958.2007.05618.x

Jeltsch, A. (2013). Oxygen, epigenetic signaling, and the evolution of early life. Trends Biochem. Sci. 38, 172-176. doi: 10.1016/j.tibs.2013.02.001

Kuhn, I., Peng, L., Bedu, S., and Zhang, C. C. (2000). Developmental regulation of the cell division protein FtsZ in Anabaena sp. strain PCC 7120, a cyanobacterium capable of terminal differentiation. J. Bacteriol. 182, 4640-4643. doi: 10.1128/JB.182.16.4640-4643.2000

Kuru, E., Tekkam, S., Hall, E., Brun, Y. V., and Van Nieuwenhze, M. S. (2015). Synthesis of fluorescent D-amino acids and their use for probing peptidoglycan synthesis and bacterial growth in situ. Nat. Protoc. 10, 33-52. doi: 10.1038/ nprot.2014.197

Lázaro, S., Fernández-Piñas, F., Fernández-Valiente, E., Blanco-Rivero, A., and Leganés, F. (2001). pbpB, a gene coding for a putative penicillin-binding protein, is required for aerobic nitrogen fixation in the cyanobacterium Anabaena sp. strain PCC7120. J. Bacteriol. 183, 628-636. doi: 10.1128/JB.183.2.628-636.2001

Lehner, J., Berendt, S., Dörsam, B., Pérez, R., Forchhammer, K., and Maldener, I. (2013). Prokaryotic multicellularity: a nanopore array for bacterial cell communication. FASEB J. 27, 2293-2300. doi: 10.1096/fj.12-225854

Lehner, J., Zhang, Y., Berendt, S., Rasse, T. M., Forchhammer, K., and Maldener, I. (2011). The morphogene AmiC2 is pivotal for multicellular development in the cyanobacterium Nostoc punctiforme. Mol. Microbiol. 79, 1655-1669. doi: 10.1111/j.1365-2958.2011.07554.x

Marbouty, M., Saguez, C., Cassier-Chauvat, C., and Chauvat, F. (2009). Characterization of the FtsZ-interacting septal proteins SepF and Ftn6 in the spherical-celled cyanobacterium Synechocystis strain PCC 6803. J. Bacteriol. 191, 6178-6185. doi: 10.1128/JB.00723-09

Mariscal, V., Nürnberg, D. J., Herrero, A., Mullineaux, C. W., and Flores, E. (2016). Overexpression of SepJ alters septal morphology and heterocyst pattern regulated by diffusible signals in Anabaena. Mol. Microbiol. 101, 968-981. doi: $10.1111 / \mathrm{mmi} .13436$

Nicolaisen, K., Hahn, A., and Schleiff, E. (2009). The cell wall in heterocyst formation by Anabaena sp. PCC 7120. J. Basic Microbiol. 49, 5-24. doi: 10.1002/ jobm. 200800300

Ponce-Toledo, R. I., Deschamps, P., López-García, P., Zivanovic, Y., Benzerara, K., and Moreira, D. (2017). An early-branching freshwater cyanobacterium at the origin of plastids. Curr. Biol. 27, 386-391. doi: 10.1016/j.cub.2016.11.056 
Prentki, P., and Krisch, H. M. (1984). In vitro insertional mutagenesis with a selectable DNA fragment. Gene 29, 303-313. doi: 10.1016/0378-1119(84)90 059-3

Randich, A. M., and Brun, Y. V. (2015). Molecular mechanisms for the evolution of bacterial morphologies and growth modes. Front. Microbiol. 6:580. doi: 10 . 3389/fmicb.2015.00580

Rudolf, M., Tetik, N., Ramos-León, F., Flinner, N., Ngo, G., Stevanovic, M., et al. (2015). The peptidoglycan-binding protein SjcF1 influences septal junction function and channel formation in the filamentous cyanobacterium Anabaena. mBio 6:e00376-15. doi: 10.1128/mBio.00376-15

Sakr, S., Jeanjean, R., Zhang, C. C., and Arcondeguy, T. (2006). Inhibition of cell division suppresses heterocyst development in Anabaena sp. strain PCC 7120. J. Bacteriol. 188, 1396-1404. doi: 10.1128/JB.188.4.1396-1404.2006

Schneider, C. A., Rasband, W. S., and Eliceiri, K. W. (2012). NIH Image to ImageJ: 25 years of image analysis. Nat. Methods 9, 671-675. doi: 10.1038/nmeth. 2089

Stanier, R. Y., Kunisawa, R., Mandel, M., and Cohen-Bazire, G. (1971). Purification and properties of unicellular blue-green algae (order Chroococcales). Bacteriol. Rev. 35, 171-205.

Tiyanont, K., Doan, T., Lazarus, M. B., Fang, X., Rudner, D. Z., and Walker, S. (2006). Imaging peptidoglycan biosynthesis in Bacillus subtilis with fluorescent antibiotics. Proc. Natl. Acad. Sci. U.S.A. 103, 11033-11038. doi: 10.1073/pnas. 0600829103

Videau, P., Rivers, O. S., Ushijima, B., Oshiro, R. T., Kim, M. J., Philmus, B., et al. (2016). Mutation of the murC and murB genes impairs heterocyst differentiation in Anabaena sp. strain PCC 7120. J. Bacteriol. 198, 1196-1206. doi: 10.1128/JB.01027-15

Wang, Y., and $\mathrm{Xu}, \mathrm{X}$. (2005). Regulation by hetC of genes required for heterocyst differentiation and cell division in Anabaena sp. strain PCC 7120. J. Bacteriol. 187, 8489-8493. doi: 10.1128/JB.187.24.8489-8493. 2005
Wolk, C. P. (1996). Heterocyst formation. Annu. Rev. Genet. 30, 59-78. doi: 10. 1146/annurev.genet.30.1.59

Yang, X., Lyu, Z., Miguel, A., McQuillen, R., Huang, K. C., and Xiao, J. (2017). GTPase activity-coupled treadmilling of the bacterial tubulin FtsZ organizes septal cell wall synthesis. Science 355, 744-747. doi: 10.1126/science.aak9995

Zhang, C. C., Huguenin, S., and Friry, A. (1995). Analysis of genes encoding the cell division protein FtsZ and a glutathione synthetase homologue in the cyanobacterium Anabaena sp. PCC 7120. Res. Microbiol. 146, 445-455.

Zhang, C. C., Laurent, S., Sakr, S., Peng, L., and Bédu, S. (2006). Heterocyst differentiation and pattern formation in cyanobacteria: a chorus of signals. Mol. Microbiol. 59, 367-375. doi: 10.1111/j.1365-2958.2005.04979.x

Zheng, Z., Omairi-Nasser, A., Li, X., Dong, C., Lin, Y., Haselkorn, R., et al. (2017). An amidase is required for proper intercellular communication in the filamentous cyanobacterium Anabaena sp. PCC 7120. Proc. Natl. Acad. Sci. U.S.A. 114, E1405-E1412. doi: 10.1073/pnas.1621424114

Zhu, J., Jäger, K., Black, T., Zarka, K., Koksharova, O., and Wolk, C. P. (2001). HcwA, an autolysin, is required for heterocyst maturation in Anabaena sp. strain PCC 7120. J. Bacteriol. 183, 6841-6851. doi: 10.1128/JB.183.23.68416851.2001

Conflict of Interest Statement: The authors declare that the research was conducted in the absence of any commercial or financial relationships that could be construed as a potential conflict of interest.

Copyright (c) 2018 Zhang, Lin, Xing and Zhang. This is an open-access article distributed under the terms of the Creative Commons Attribution License (CC BY). The use, distribution or reproduction in other forums is permitted, provided the original author(s) and the copyright owner are credited and that the original publication in this journal is cited, in accordance with accepted academic practice. No use, distribution or reproduction is permitted which does not comply with these terms. 